\title{
Differential Serum Levels of Eosinophilic Eotaxins in Primary Sclerosing Cholangitis, Primary Biliary Cirrhosis, and Autoimmune Hepatitis
}

\author{
Abdolamir Landi, ${ }^{1}$ Tobias J. Weismuller, ${ }^{2,3}$ Tim O. Lankisch, ${ }^{2}$ Deanna M. Santer, \\ D. Lorne J. Tyrrell, ${ }^{1}$ Michael P. Manns, ${ }^{2}$ and Michael Houghton ${ }^{1}$
}

To investigate pathogenic mechanisms of primary sclerosing cholangitis (PSC), primary biliary cirrhosis (PBC), and autoimmune hepatitis (AIH), serum levels of 26 chemokines and cytokines were determined and compared with patients with chronic hepatitis $\mathrm{C}$ or in healthy controls.

The chemokine eotaxin-3 (E3; CCL26), which recruits eosinophils to sites of inflammation, was found to be highly elevated in all PSC, PBC, and AIH patients compared with HCV patients and healthy controls. Eotaxin-1 (E1; CCL11), another eosinophil-specific chemokine, was elevated in PSC but reduced in PBC and AIH, while the macrophage-derived chemokine (MDC; CCL22) was lower in all PSC, PBC, and AIH patients compared with HCV patients and controls. By incorporating levels of the interleukin (IL)-15 into a diagnostic algorithm, PSC, PBC, and AIH patients could each be differentiated with good sensitivity and specificity. These findings represent the first study to compare the level of serum cytokine/chemokine levels among these related autoimmune-like liver diseases. Furthermore, our data indicate that the measurement of serum E3, E1, CCL22, and IL-15 levels can aid in the diagnosis of these clinically challenging diseases and shed light on the potential pathogenic mechanisms underlying these diseases. By suggesting a potential role for an allergic phenomenon involving eosinophils, which may define them as liver-specific allergic diseases, this may open up potential new therapeutic avenues by abrogating the action of these disease-associated immune modulators.

\section{Introduction}

$\mathrm{P}_{\mathrm{s}}^{\mathrm{n}}$ RIMARY SCLEROSING CHOLANGITIS (PSC), primary biliary cirrhosis (PBC), and autoimmune hepatitis (AIH) are 3 distinct chronic inflammatory liver diseases. The causes of these diseases are unknown, but they are generally viewed as autoimmune diseases of the liver, although an infectious etiology is possible for at least some of them. Their onset is usually associated with nonspecific symptoms of liver diseases, such as fatigue, right upper quadrant abdominal pain, nausea, jaundice, and/or pruritus along with fluctuating nonspecific elevations of liver enzymes that indicate the presence of liver inflammation (Boyer and others 2011). However, liver enzyme elevations often occur at later stages of the disease when fibrosis and cirrhosis is present.

The pathology of AIH starts with damage to the hepatocytes resulting in interface hepatitis along with infiltration of leukocytes eventually followed by fibrosis and cirrhosis (Lohse and Mieli-Vergani 2011). In PBC, which is described as a chronic nonsuppurative destructive granulomatous cholangitis of unknown etiology, the pathology centers on small- to medium-sized $(<100 \mu \mathrm{m})$ intrahepatic bile ducts rather than hepatocytes, resulting in cholestatic features of the disease, which can sometimes, but not always, be associated with a high level of alkaline phosphatase (AP) in serum (Kaplan and Gershwin 2005). As in AIH, PBC is more common in women and is considered a liver-specific autoimmune disease occurring in genetically predisposed individuals in association with other autoimmune conditions, such as Sjogren's syndrome. However, unlike other autoimmune diseases, PBC has not been reported in children. Similar to PBC, PSC is also a chronic cholestatic condition, but it affects all sizes of intra/extrahepatic bile ducts ultimately resulting in fibrosis and cirrhosis (Angulo and Lindor 1999a). PSC is more common in men and is highly associated with inflammatory bowel diseases (IBDs), especially ulcerative colitis, which is reported in more than $60 \%$ of PSC cases (Tischendorf and others 2007). In both PBC and PSC, inflammation usually starts around or close to the biliary system resulting in the cholestatic features of the disease;

\footnotetext{
${ }^{1}$ Department of Medical Microbiology and Immunology, Li Ka Shing Institute of Virology, University of Alberta, Edmonton, Canada.

${ }^{2}$ Department of Gastroenterology, Hepatology and Endocrinology, Medical School of Hannover, Hannover, Germany.

${ }^{3}$ Department of Internal Medicine 1, University of Bonn, Bonn, Germany.
} 
however, the pathology in PBC results from destruction of the biliary ducts, whereas in PSC, fibrosis initiates and progresses within areas surrounding the bile ducts. In contrast to AIH and PBC, males are affected more often than females; PSC is also characterized by an $80 \%$ association with IBD, as well as biliary and extrahepatic malignancies.

Currently, there is no specific autoantibody, immunologic, biochemical, or serological diagnostic biomarker for diagnosis of PSC (Boyer and others 2011). Histology obtained from liver biopsy is usually not helpful in this bile duct disease because of its focal nature. Diagnosis is mainly based on endoscopic retrograde cholangiography (ERC) or magnetic resonance cholangiography (MRC). The disease is often confirmed in late and irreversible stages of the illness as it is based on the detection of typical strictures and dilations in intra/extrahepatic bile ducts due to progressed concentric fibrosis. In addition, imaging is invasive (ERC) with the risk of complications, such as pancreatitis, bacterial cholangitis, perforation, and bleeding or suboptimal (in the case of MRC) in the detection of early intrahepatic bile duct disease and cirrhosis. In the case of AIH, the International Autoimmune Hepatitis Group has recently suggested a simplified version (Lohse and Mieli-Vergani 2011) of a previously described algorithm for the diagnosis. However, in this 4-parameter algorithm after the exclusion of viral hepatitis, the detection of at least one associated autoantibody and elevated levels of total IgG, there is still a need for liver biopsy to confirm the diagnosis. Because of the risks inherent in liver biopsy (Burak and others 2003), this may not be performed in which case diagnosis is confirmed only after observing a response to immunosuppressive treatment. For PBC, there is a highly sensitive (90\%-95\%) and specific (100\%) serological test based on the detection of antimitochondria antibody (AMA) against the E2 subunit of the pyruvate dehydrogenase located on the inner mitochondrial membrane along with elevated levels of serum IgM (Kaplan and Gershwin 2005). However, liver biopsy is still required to diagnose AMAnegative cases of PBC.

In brief, the methods of diagnosis for these disorders are suboptimal and usually provide a diagnosis only at later stages of liver disease when there is often substantial and irreversible liver pathology.

Although AIH, PSC, and PBC are currently considered manifestations of autoimmunity, a role for viral infection has not been ruled out (Minuk and others 1987; Vento and Cainelli 2004; Mason 2011). To explore potential immune mediators of these diseases, we determined the levels of cytokines and chemokines in the peripheral blood of AIH, PBC, and PSC patients and compared them with those in patients with liver disease known to be caused by a chronic viral infection and in healthy controls.

\section{Materials and Methods}

\section{Cohorts}

Hepatitis C patients were diagnosed using commercial serology and polymerase chain reaction assays and were positive for the virus at the time of recruitment. They had either not received any treatment or not responded to the standard treatment. None of the treated patients had received any treatment for at least 1 year. All HCV patients were negative for HBV and HIV. Healthy individuals were negative for HCV, HBV, and HIV. Serum samples from PSC (with/without IBD), PBC, and AIH patients were from the Hannover Medical School in Germany. The diagnosis of PSC was based on the typical cholangiographic findings, such as strictures or irregularities of intra- or extrahepatic bile ducts and exclusion of secondary causes for sclerosing cholangitis. PBC was diagnosed by positive finding of AMAs against E2 subunit of pyruvate dehydrogenase (PDH-E2), cholestatic liver diseases, and typical histology. $\mathrm{AIH}$ was diagnosed by defined clinical, biochemical, histology criteria, and autoantibody parameters (Manns and others 2010). The subjects' characteristics are summarized in Table 1. Informed consent was obtained from each participant included in the study, and the study protocol conforms to the ethical guidelines of the 1975 Declaration of Helsinki as reflected in a priori approval by the Human Research Ethics Boards of the University of Alberta and Hannover Medical School.

\section{Serum collection}

Venous blood was drawn from subjects by venipuncture using standardized phlebotomy procedures into gold-top vacutainer tubes (Becton Dickinson, Franklin Lakes, NJ). The sera were aspirated from the top of the centrifuged coagulated blood and stored at $-80^{\circ} \mathrm{C}$ until use.

\section{Analysis of cytokine/chemokine levels in serum}

After comparing 3 different multiplex technologies, the Human Multiplex Ultra-Sensitive Kit from Meso Scale Discovery (MSD, Gaithersburg, MA) was preferred because of higher sensitivity, lower level of detection, and wide dynamic range with the standard curve being linear within the range of experimental samples as previously described (Fichorova and others 2008). All experiments were performed according to the manufacturer's instruction with minimal modifications and optimization. Plates were finally scanned with a SECTOR ${ }^{\circledR}$ Imager 6000 Reader (MSD), results were analyzed for validity, precision, and accuracy, and obtained concentrations were corrected for dilution.

Table 1. Number, Sex, and Age of Subjects in Study

\begin{tabular}{lccccc}
\hline & Controls & HCV & PSC & PBC & AIH \\
\hline Number of subjects & 50 & 54 & 58 & 50 & 40 \\
Sex (M/F) & $27 / 23$ & $29 / 25$ & $39 / 19$ & $6 / 44$ & $18 / 22$ \\
Age (mean \pm SD) & $50.3 \pm 8.2$ & $52.3 \pm 10.5$ & $46.2 \pm 11.1$ & $56.1 \pm 12.7$ & $46.6 \pm 18.6$ \\
\hline
\end{tabular}

Controls, healthy controls; HCV, chronic hepatitis C; PSC, primary sclerosing cholangitis; PBC, primary biliary cirrhosis; $\mathrm{AIH}$, autoimmune hepatitis; SD, standard deviation; $\mathrm{M}$, male; F, female. 


\section{Statistical analysis}

Data were analyzed using statistical software (GraphPad Prism Version 5.00; Prism Software Corporation, Irvine, CA). As outcome variables from each group were not distributed normally, differences among all groups were examined using the nonparametric Kruskal-Wallis test with the Dunn's posttest. If a significant difference was found among the groups, the medians between each pairs of groups were compared using the Mann-Whitney $U$-test. Differences were consid-
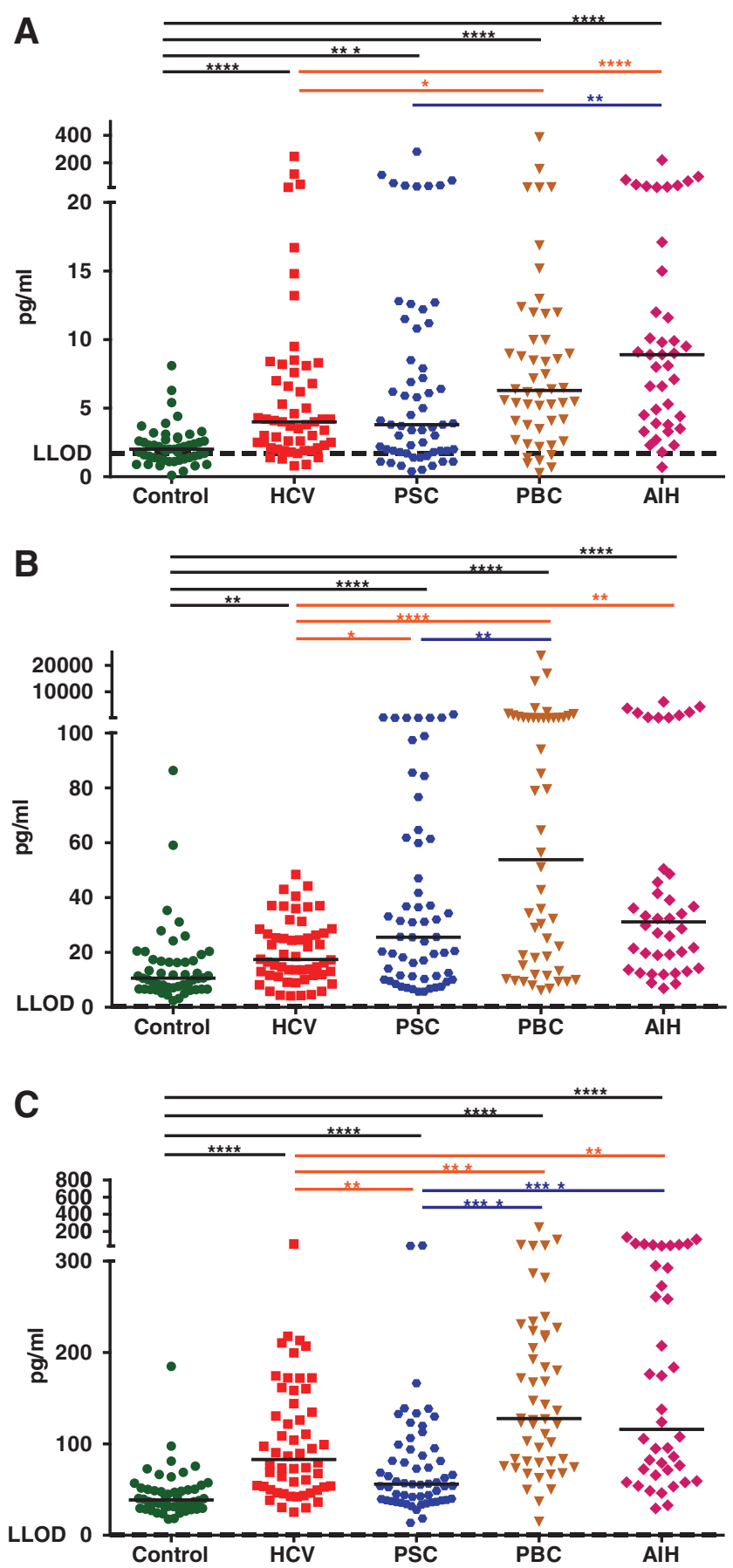

ered significant if the 2-tailed $P$ value was lower than 0.05 with confidence intervals of $95 \%$.

\section{Results \\ Cytokine/chemokine profile in chronic hepatitis $C$ patients}

In the HCV group, significant elevations were observed in the levels of interleukin (IL)-6, IL-8, monokine induced by
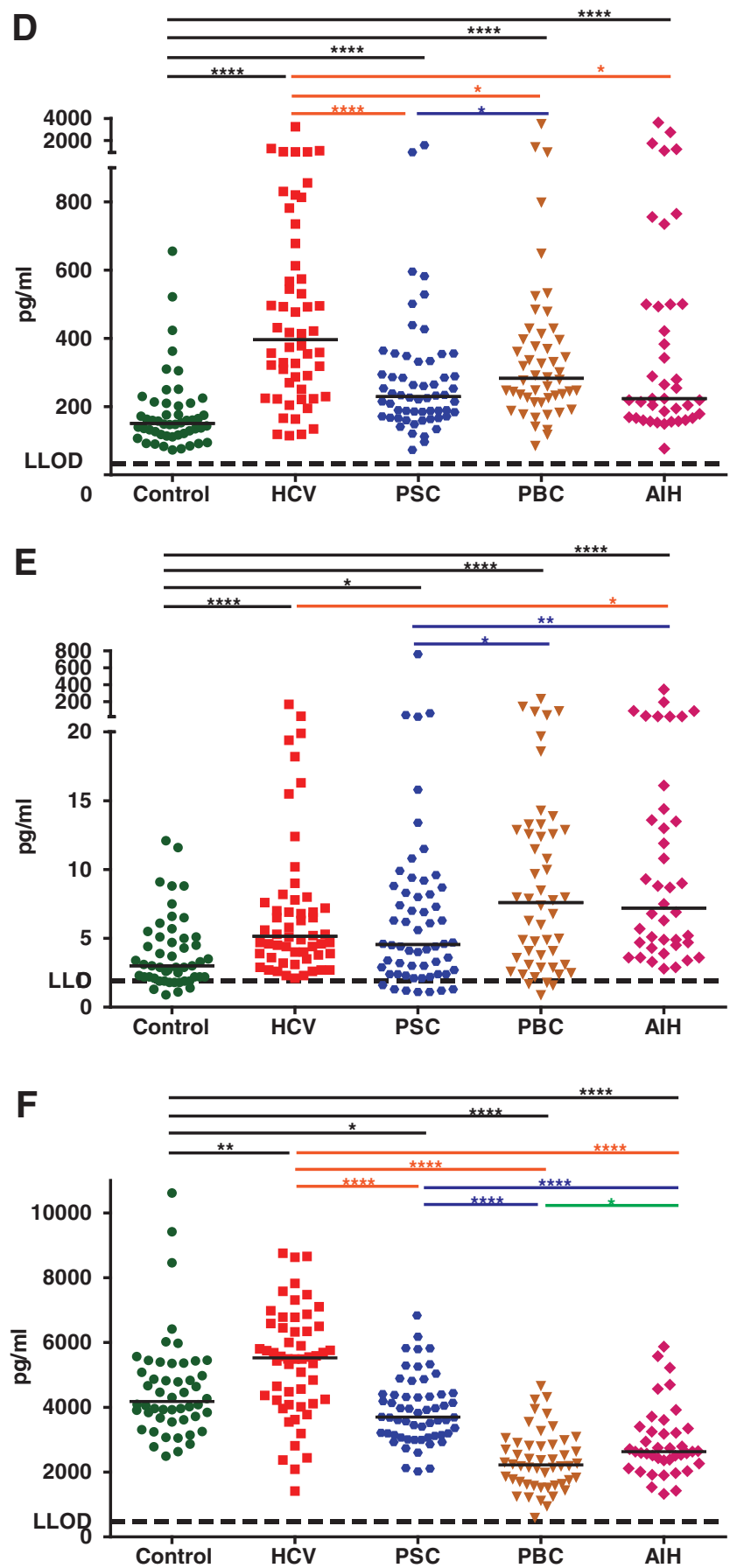

FIG. 1. The level of cytokines/chemokines in sera of the study groups. (A) IL-6. (B) IL-8. (C) CXCL9. (D) CXCL10. (E) IL-10. (F) CCL22. Short horizontal lines in each group indicate median values. The asterisk symbols $\left({ }^{*}, * *, * *\right.$, and $\left.{ }^{* * *}\right)$ are indicating of $P<0.05, P<0.01, P<0.001$, and $P<0.0001$, respectively. Control, healthy controls; HCV, chronic hepatitis C; PSC, primary sclerosing cholangitis; PBC, primary biliary cirrhosis; AIH, autoimmune hepatitis; LLOD, lower level of detection; IL, interleukin. 
gamma-interferon (MIG; CXCL9), interferon-gammainduced protein 10 (IP-10; CXCL10), IL-10, MDC (CCL22), and IL-15 in comparison to healthy controls (Figs. 1A-F and 2A). In contrast, significant decreases were observed in the levels of IL-7 and monocyte chemoattractant protein 4 (MCP-4; CCL13) in HCV samples compared with healthy controls (Fig. 2B, C).

\section{Cytokine/chemokine profile in PSC}

In PSC patients, the levels of IL-6, IL-8, CXCL9, CXCL10, IL-10, and macrophage inflammatory proteins 1-beta (MIP$1 \beta$; CCL4) were significantly higher than that of healthy controls (Figs. 1A-E and 2D), whereas the levels of CCL22 and IL-15 were significantly lower than that of healthy
A

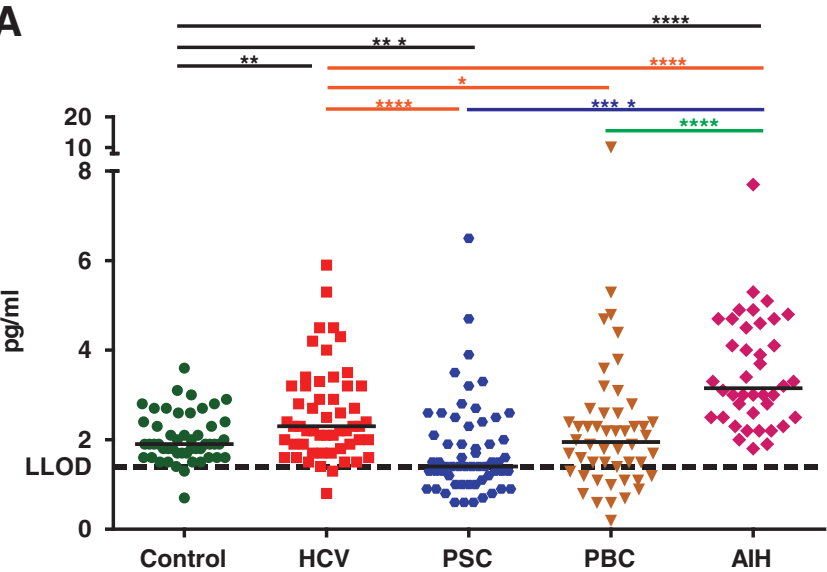

B

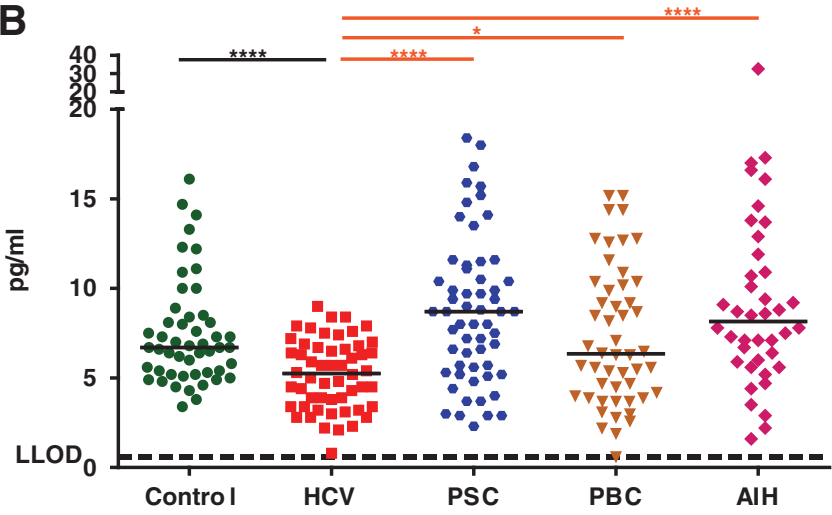

C

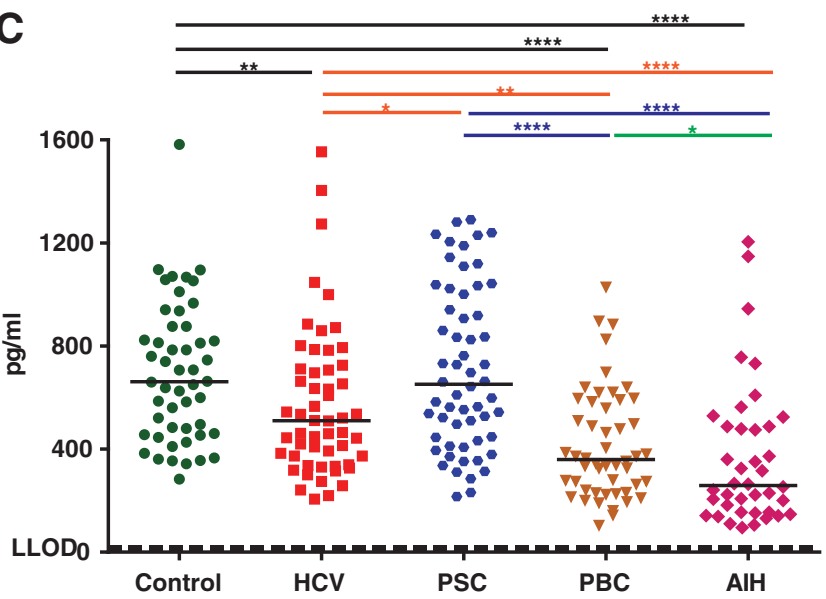

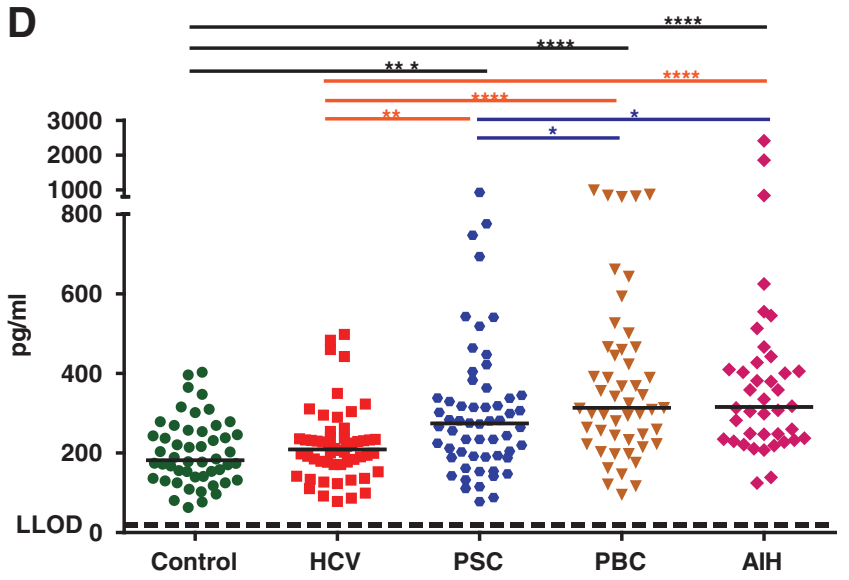

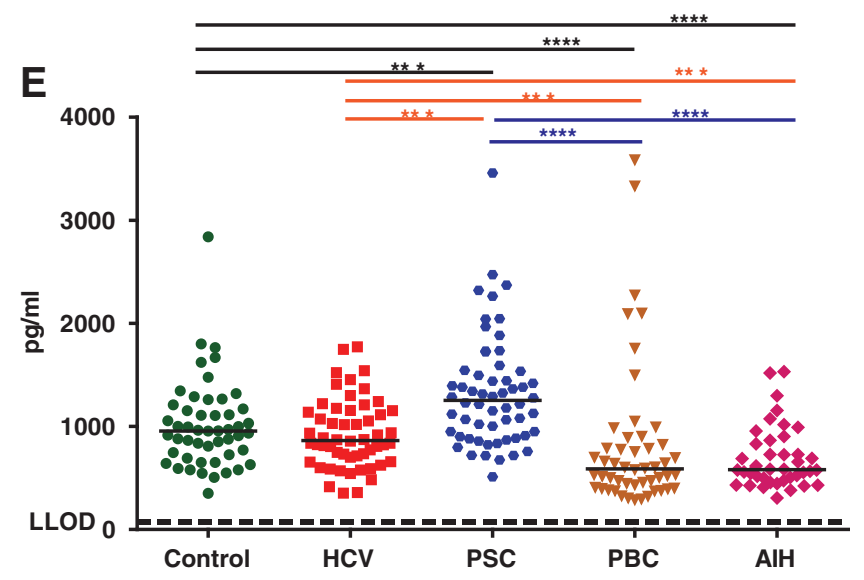

$\mathbf{F}$
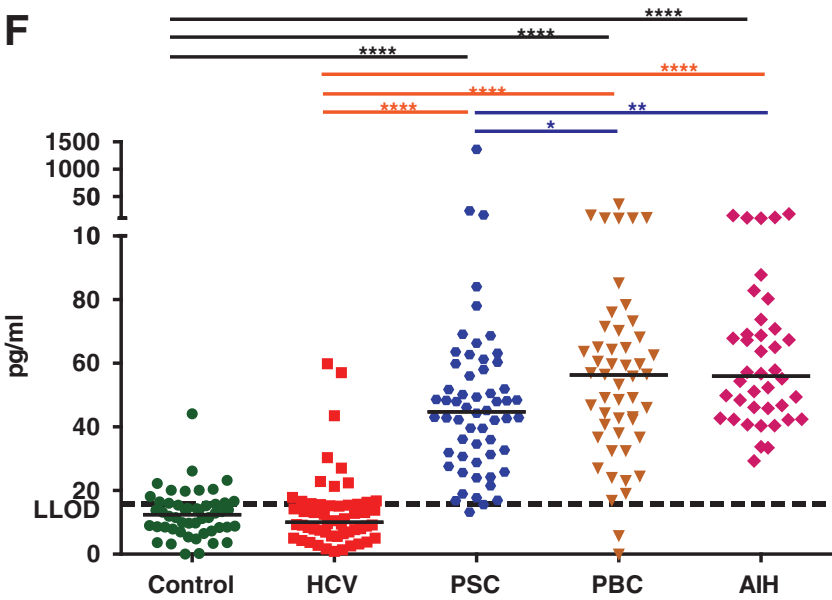

FIG. 2. The level of cytokines/chemokines in sera of the study groups. (A) IL-15. (B) IL-7. (C) CCL13. (D) CCL4. (E) E1. (F) E3. Short horizontal lines in each group indicate median values. The asterisk symbols $\left({ }^{*},{ }^{* *},{ }^{* * *}\right.$, and $\left.{ }^{* * * *}\right)$ are indicating of $P<0.05, P<0.01, P<0.001$, and $P<0.0001$, respectively. E3, eotaxin-3; E1, eotaxin-1. 
controls (Figs. 1F and 2A). Strikingly, eotaxin-1 (E1; CCL11) and particularly eotaxin-3 (E3; CCL26) levels were very high in sera of PSC patients compared with the levels in sera of $\mathrm{HCV}$ patients or healthy controls (Fig. 2E, F).

\section{Cytokine/chemokine profile in PBC}

In PBC patients, the levels of IL-6, IL-8, CXCL9, CXCL10, IL-10, CCL4, E3, interferon gamma (IFN- $\gamma$ ), IL-12 p70, IL-1 $\beta$, IL-4, IL-5, and IL-13 were significantly increased in sera compared with the levels in healthy controls. Of those, all were also higher than that of HCVs with the exception of CXCL10 that was lower and IL-10, which was not different from $\mathrm{HCV}$ patients (Figs. 1A-E, 2D, F, and 3A-F). In contrast, the levels of CCL22, CCL13, E1, tumor necrosis factor alpha (TNF- $\alpha)$, and thymus and activation regulated chemokine (CCL17) were significantly lower than that of healthy controls as well as HCV patients with the exception of CCL17 that was not significantly different from HCV patients (Figs. 1F, 2C, E, and 4A, B). In addition, while not
A

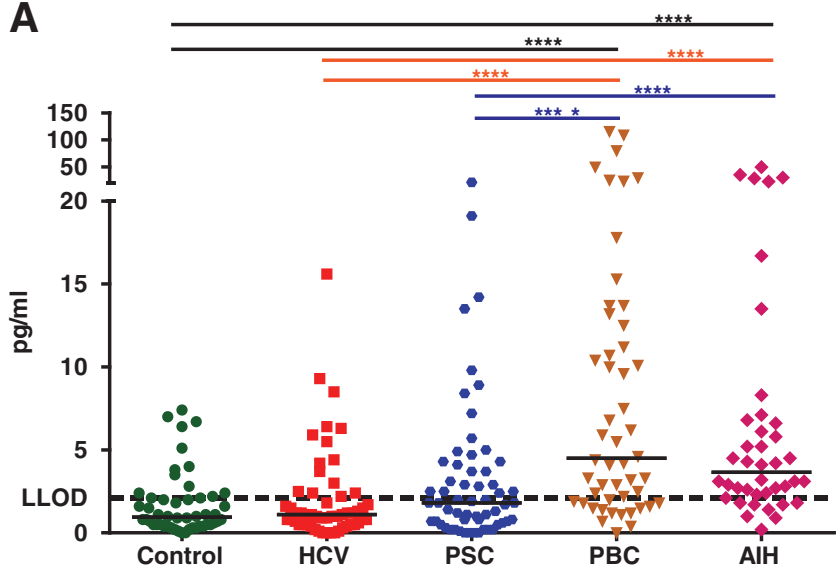

B
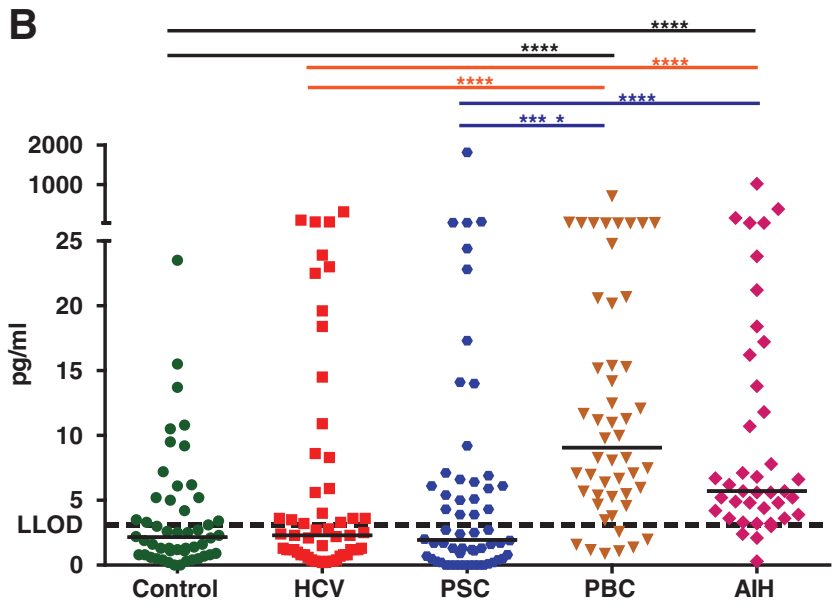

C

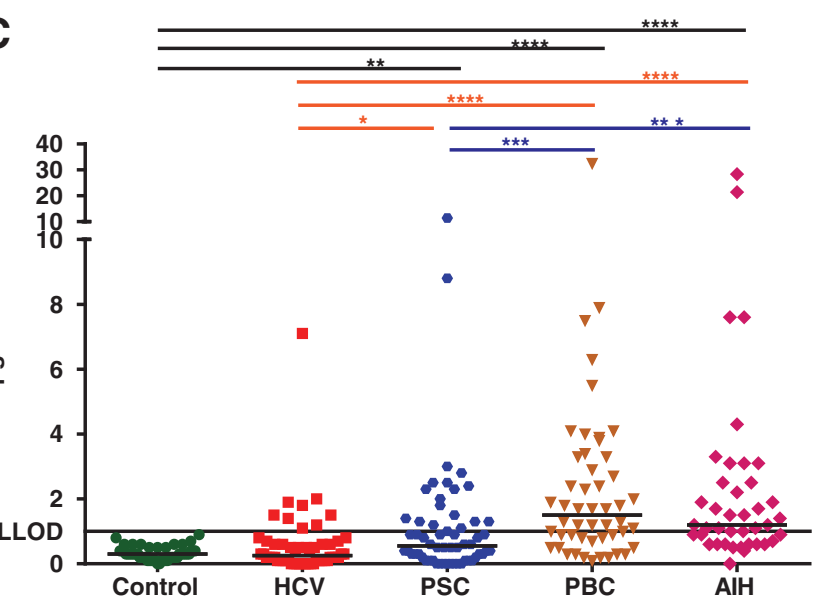

D

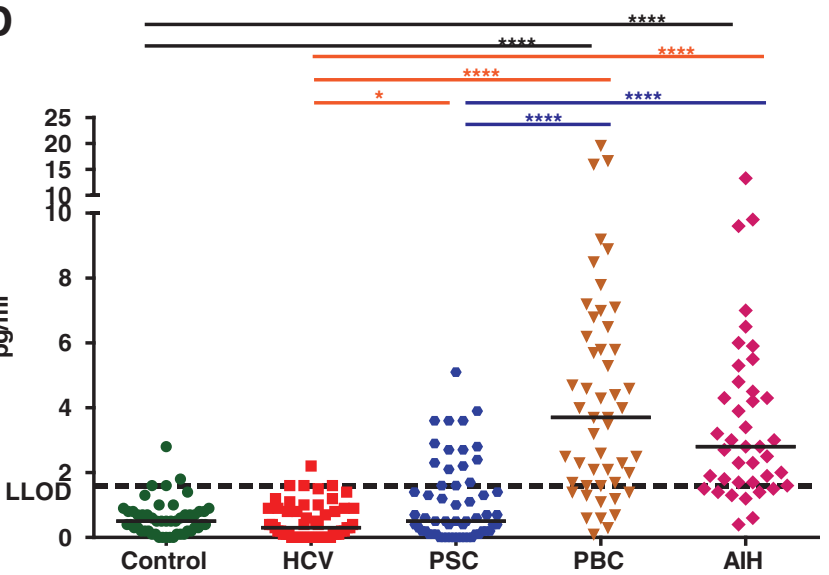

E
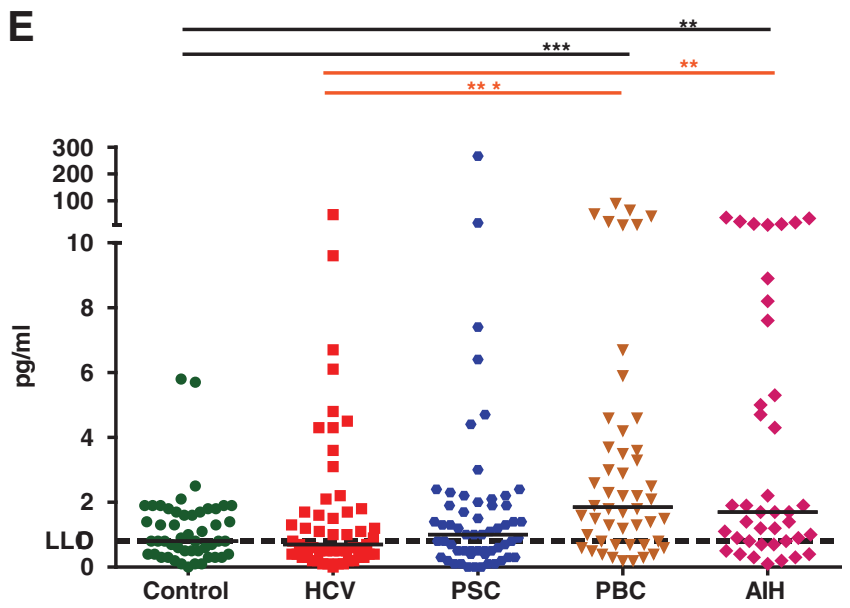

F
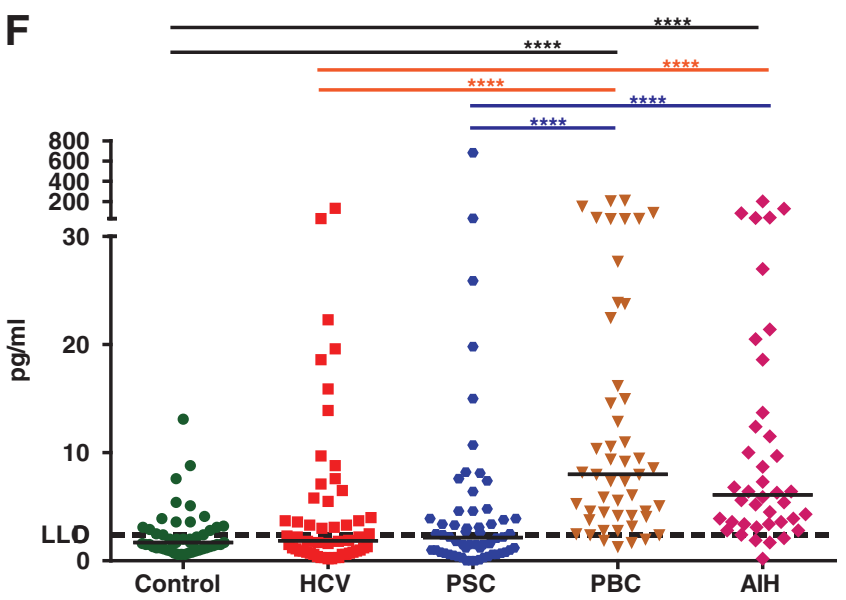

FIG. 3. The level of cytokines/chemokines in sera of the study groups. (A) IFN- $\gamma$. (B) IL-12p70. (C) IL-1ß. (D) IL-4. (E) IL-5. (F) IL-13. Short horizontal lines in each group indicate median values. The asterisk symbols $\left(^{*},{ }^{* *},{ }^{* * *}\right.$, and $\left.{ }^{* * *}\right)$ are indicating of $P<0.05, P<0.01, P<0.001$, and $P<0.0001$, respectively. IFN- $\gamma$, interferon gamma. 
A

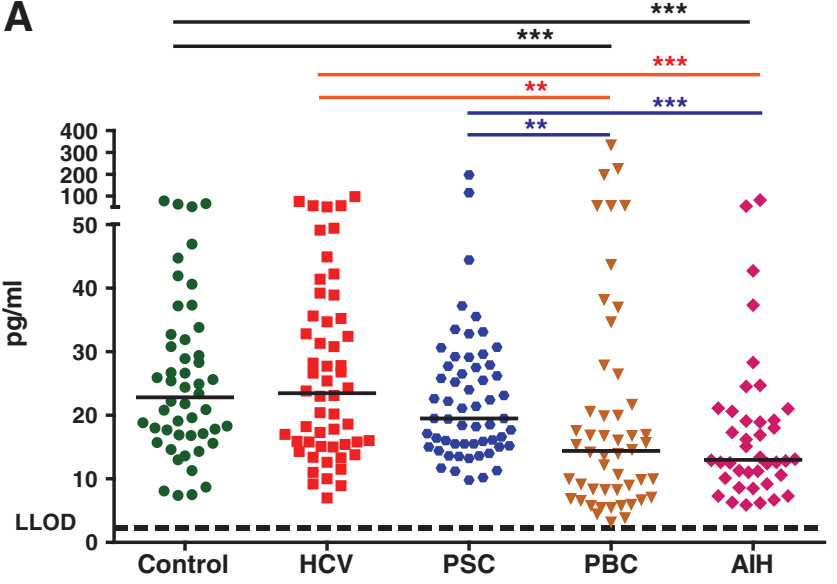

B
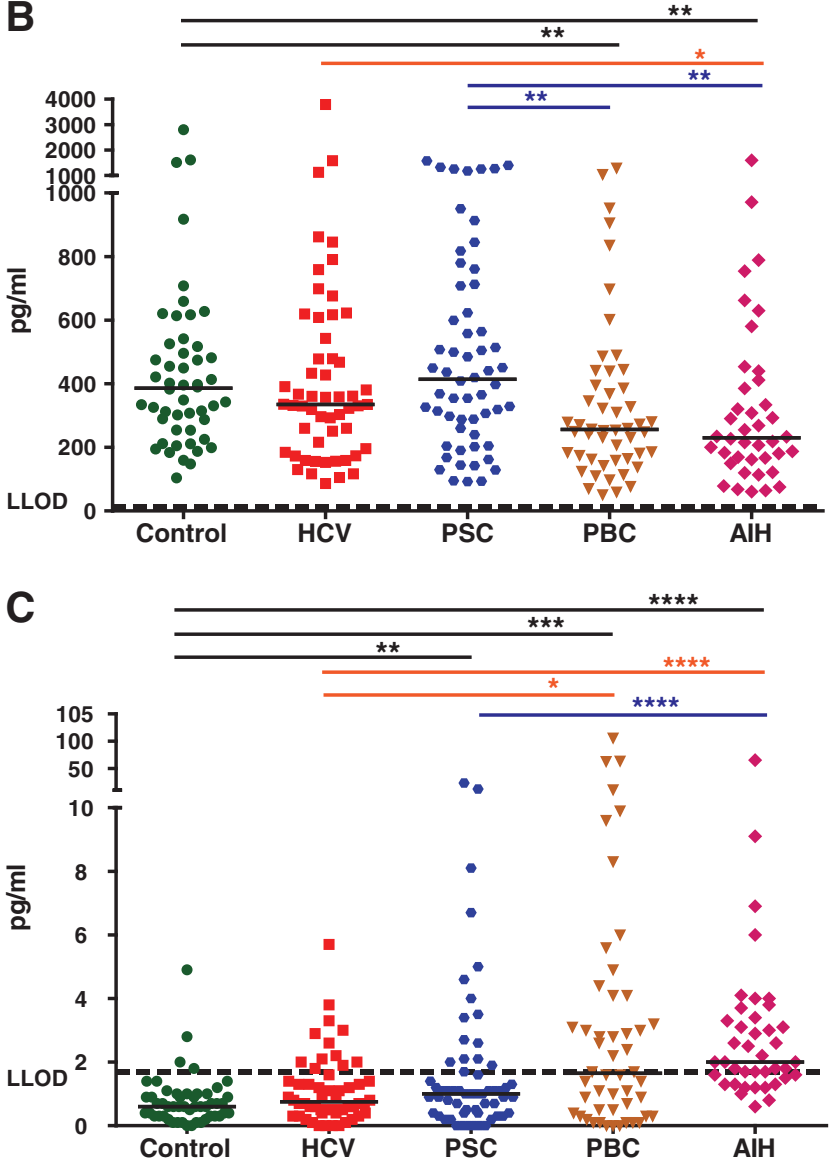

FIG. 4. The level of cytokines/chemokines in sera of the study groups. (A) TNF- $\alpha$. (B) CCL17. (C) IL-2. Short horizontal lines in each group indicate median values. The asterisk symbols $\left(*, * *, * * *\right.$, and $\left.{ }^{* * * *}\right)$ are indicating of $P<0.05$, $P<0.01, P<0.001$, and $P<0.0001$, respectively. TNF- $\alpha$, tumor necrosis factor alpha.

different from healthy controls, the level of IL-15 was lower and the level of IL-7 was higher than that of HCV patients (Fig. 2A, B).

\section{Cytokine/chemokine profile in $\mathrm{AlH}$}

The cytokine/chemokine profile in AIH was highly similar to PBC with the exception of IL-15, which was significantly elevated in $\mathrm{AIH}$ in comparison to the healthy control, HCV, PSC, and PBC groups (Fig. 2A). In addition, the level of IL-10 was higher, whereas CCL17 was lower in $\mathrm{AIH}$ patients compared with $\mathrm{HCV}$ patients (Fig. 1E and Fig. 4B).

\section{Discriminatory cytokine/chemokine profiles associated with PSC, $P B C$, and $A I H$}

The levels of E1 and E3 were strikingly higher in PSC patients than in healthy controls and HCV patients (Fig. 2E, F). In contrast, the level of IL-15 was significantly lower in PSC patients compared with healthy controls and HCV patients (Fig. 2A). When the profiles of $\mathrm{PBC}$ and $\mathrm{AIH}$ was compared to $\mathrm{HCV}$ patients, the most remarkable difference was observed in the level of E3 (but not E1), which was significantly elevated in PBC and $\mathrm{AIH}$ in comparison to that of healthy controls and HCVs (Fig. 2F). The level of CCL22 in all PSC, PBC, and AIH patients was lower than that of healthy controls and $\mathrm{HCV}$ patients (Fig. 1F). In the case of AIH, the level of IL-15 in the sera appeared to be significantly higher than that of all groups, including the HCV group (which was significantly higher than that of healthy controls; Fig. 2A). This was not the case for PBC patients, in which the level of IL-15 was no different from that of healthy controls and lower than that of the HCV patients. The cytokine/chemokine profiles of PBC and $\mathrm{AIH}$ patients were very similar with the exception of IL-15, which was significantly higher in AIH patients (Fig. 2A). This similarity was also true for a group of analytes, including CCL13, E1, TNF- $\alpha$, which were all significantly reduced in both $\mathrm{PBC}$ and $\mathrm{AIH}$ patients compared with healthy controls, HCV and PSC patients (Figs. 2C, E and 4A). A summarized list of the cytokines and chemokines that are significantly changed in any of the patient groups in comparison to the healthy controls is described in Table 2 for the reference.

\section{New biomarkers and algorithms of diagnostic value}

The level of E3 in sera is highly elevated in all PSC, PBC, and $\mathrm{AIH}$ groups in comparison to that of healthy controls and HCV patients and as such may represent an important new biomarker associated with these 3 diseases. Moreover, E1, CCL22, and IL-15 levels are significantly different when compared with healthy controls and HCV patients. The intra- and interassay coefficient variances for each analyte were $10 \%$ and $12 \%$ for $\mathrm{E} 1,12 \%$ and $9 \%$ for $\mathrm{E} 3,9 \%$ and $8 \%$ for CCL22, and $15 \%$ and $5 \%$ for IL-15, respectively, indicating the high reproducibility of the data. By constructing receiveroperating characteristic (ROC) curves, we determined cutoff levels for E3, E1/E3 ratio, CCL22, and IL-15 that were able to discriminate PSC, PBC, and AIH groups from healthy control and HCV cases and from each other (Fig. 5). Using a cutoff value of E3 $>28 \mathrm{pg} / \mathrm{mL}$, PSC, PBC, and AIH can be distinguished from healthy controls with a sensitivity of $79 \%$, $84 \%$, and $100 \%$, respectively, with low positivities of $2 \%$ and $7 \%$ in healthy controls and HCV patients, respectively (Table 3). We calculated that determining elevations in E3 serum levels in addition to the current routine test for elevated levels of AP (AP $\uparrow)$ increases the detection rate for PSC patients from $55 \%$ to $97 \%$ (Fig. 6). In addition, by determining the E1/E3 ratio as well as the circulating CCL22 level, we 
Table 2. The Summarized List of Cytokines/Chemokines with Significant Changes in Patients' Groups in Comparison to the Healthy Control Group

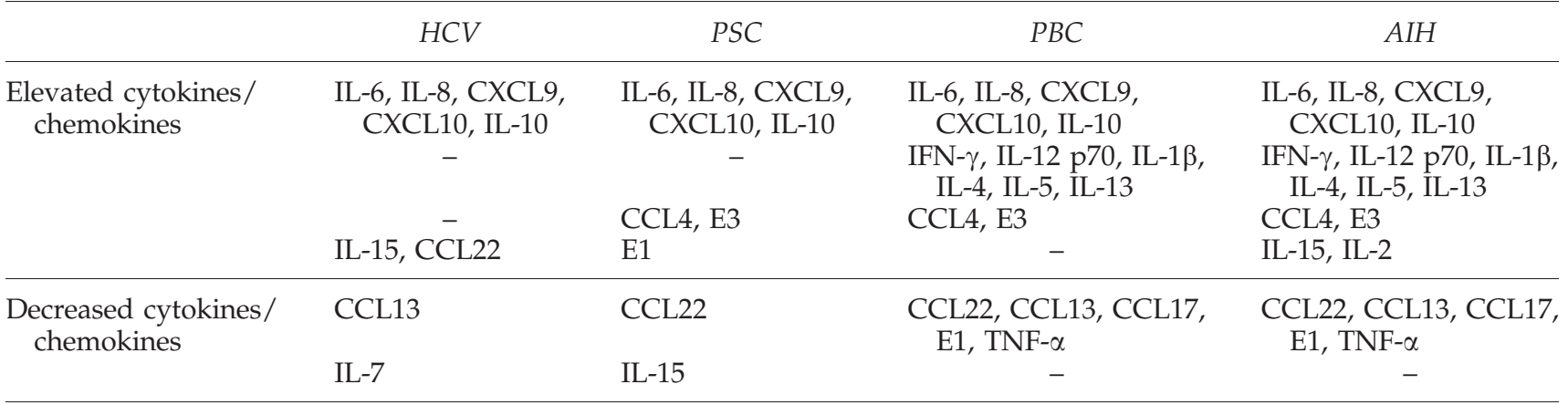

The cytokines and chemokines are presented in several rows for easier comparison among groups.

E3, eotaxin-3; E1, eotaxin-1; IL, interleukin; IFN- $\gamma$, interferon gamma; TNF- $\alpha$, tumor necrosis factor alpha.

A
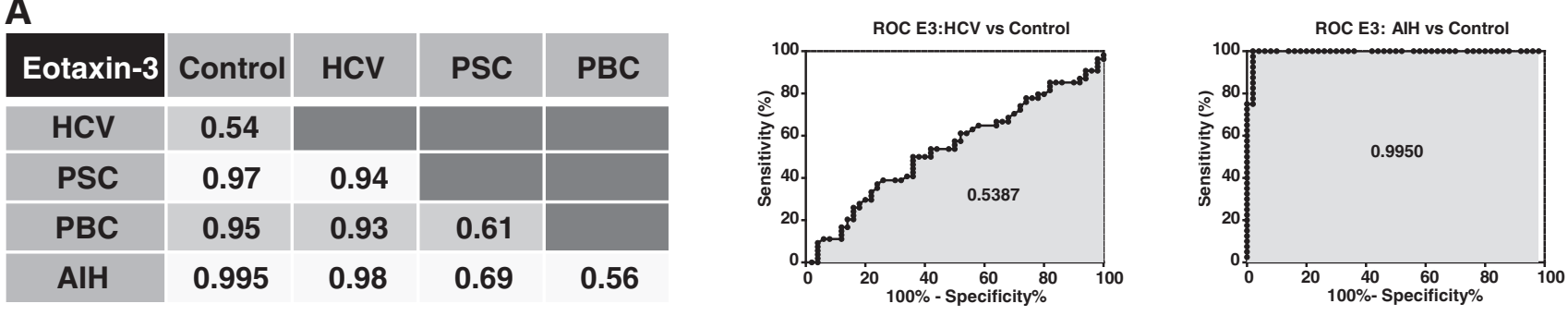

B

\begin{tabular}{|c|c|c|c|c|}
\hline E1/E3 & Control & HCV & PSC & PBC \\
\hline HCV & 0.52 & & & \\
\hline PSC & 0.91 & 0.89 & & \\
\hline PBC & 0.94 & 0.93 & 0.81 & \\
\hline AlH & 0.999 & 0.98 & 0.91 & 0.54 \\
\hline
\end{tabular}
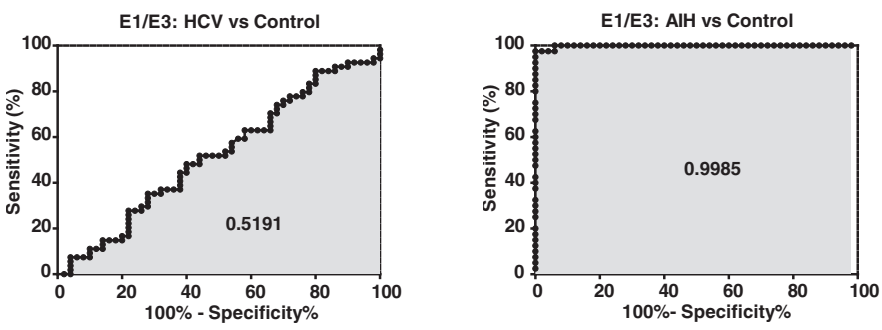

C

\begin{tabular}{|c|c|c|c|c|}
\hline CCL22 & Control & HCV & PSC & PBC \\
\hline HCV & 0.69 & & & \\
\hline PSC & 0.64 & 0.78 & & \\
\hline PBC & 0.93 & 0.94 & 0.88 & \\
\hline AlH & 0.85 & 0.88 & 0.78 & 0.66 \\
\hline
\end{tabular}
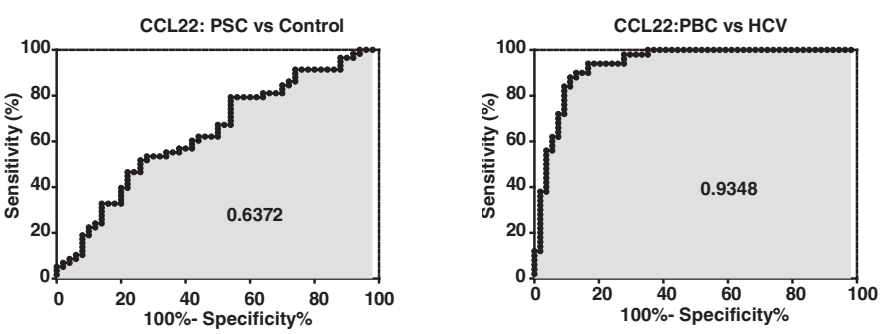

D

\begin{tabular}{|c|c|c|c|c|}
\hline IL-15 & Control & HCV & PSC & PBC \\
\hline HCV & 0.66 & & & \\
\hline PSC & 0.69 & 0.76 & & \\
\hline PBC & 0.52 & 0.63 & 0.61 & \\
\hline AlH & 0.89 & 0.74 & 0.89 & 0.81 \\
\hline
\end{tabular}
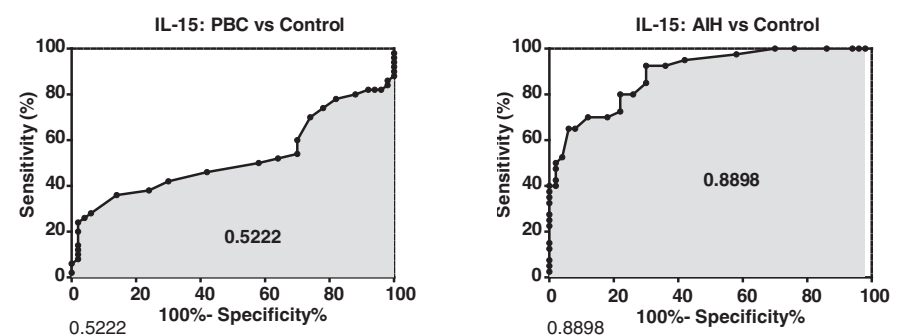

FIG. 5. The use of ROC curves and AUC values in evaluation of the sensitivity and specificity of the diagnostic biomarkers. (A) E3. (B) E1/E3 ratio. (C) CCL22. (D) IL-15. Numbers within the tables/curves are AUC values for any 2 correspondent clinical conditions. The plots in the middle and right columns show representative curves for the lowest and highest AUC in the correspondent group, respectively. An AUC value of 1 is indicative of $100 \%$ sensitivity and specificity (a perfect test), and a value of 0.5 is indicative of $50 \%$ sensitivity and specificity (an entirely worthless test). The horizontal axes on the curves are presenting $100 \%$ minus specificity (the highest specificity of $100 \%$ is located at zero). ROC, receiver-operating characteristic curves; AUC, area under the curve. 
Table 3. The Sensitivity and Specificity of Suggested Diagnostic Biomarkers/Algorithms

\begin{tabular}{|c|c|c|c|c|c|c|c|}
\hline \multirow[b]{2}{*}{ Facilitated diagnosis } & \multirow[b]{2}{*}{ Biomarker/algorithm } & \multirow[b]{2}{*}{ Sensitivity (\%) } & \multicolumn{5}{|c|}{ Specificity (\%) to: } \\
\hline & & & Ctrl & $\mathrm{HCV}$ & PSC & $P B C$ & $A I H$ \\
\hline PSC, PBC, or AIH & E3 $>28$ & 79,84, or 100 & 98 & 93 & NA & NA & NA \\
\hline PSC & $\mathrm{E} 3>28+\mathrm{E} 1 / \mathrm{E} 3>15+\mathrm{CCL} 22>2800$ & 67 & 98 & 98 & NA & 94 & 92 \\
\hline PSC & $\mathrm{E} 3>25+\mathrm{E} 1 / \mathrm{E} 3>15+\mathrm{CCL} 22>2800$ & 72 & 97 & 94 & NA & 94 & 92 \\
\hline PSC & $\mathrm{E} 3>23+\mathrm{E} 1 / \mathrm{E} 3>15+\mathrm{CCL} 22>2800$ & 76 & 94 & 94 & NA & 90 & 92 \\
\hline $\mathrm{AIH}$ & $\mathrm{E} 3>25+\mathrm{E} 1 / \mathrm{E} 3<15+\mathrm{CCL} 22>1870+\mathrm{IL}-15>2.4$ & 60 & 100 & 100 & 100 & 90 & NA \\
\hline
\end{tabular}

Ctrl, healthy controls; NA, not applicable.

derived 2 algorithms that could discriminate PSC (sensitivity of $67 \%-76 \%)$ from healthy controls $(2 \%-6 \%)$, HCV $(4 \%-6 \%)$, PBC (6\%-10\%), and AIH (8\%; Table 3). Finally, we derived another algorithm based on the concentrations of E1, E3, CCL22, and IL-15 to enable the discrimination of AIH (with $60 \%$ sensitivity) from healthy controls ( $0 \%)$, HCV (0\%), PSC $(0 \%)$, and PBC (10\%; Table 3$)$.

\section{Discussion}

This is the first study to determine the cytokine/chemokine profiles of these challenging AILDs from which it was possible to distinguish them from each other and from $\mathrm{HCV}$ patients and healthy controls. Strikingly, we found highly elevated levels of E3 in these patients. Some similarities were observed between HCV and AILD patients such as elevated levels of the proinflammatory cytokines IL- 6 and IL- 8 and the regulatory cytokine, IL-10, which could be consistent with a possible infectious aetiology for PSC, PBC, and AIH. In addition, elevated levels of IFN- $\gamma$, IL-12p70, IL5 , and IL-1 $\beta$ were observed in AIH and PBC, typical of a Th1-skewed state of immune activation, which is also consistent with an infectious aetiology. Furthermore, AIH was found to be associated with elevated levels of IL-15, an antiviral Th1-type cytokine, which induces T-cell proliferation, production of IFN- $\gamma$, and TNF- $\alpha$ by macrophages and plays an essential role in proliferation and survival of NK cells (Ranson and others 2003). Although not definitive, these observations support speculation that one or more of these autoimmune liver diseases may have an infectious etiology, a speculation, which could guide new research directions.
Our results demonstrated statistically significant differences in the circulating concentrations of E3, E1, CCL22, and IL-15 among the various patient groups. These differences were evaluated by constructing ROC curves, the high sensitivities and specificities of which indicate strong diagnostic potential (Fig. 5). Serum E3 levels can be used as a biomarker to discriminate PSC, PBC, and AIH from healthy controls and HCV patients with a sensitivity of $79 \%-100 \%$ and a high specificity of $97 \%-98 \%$ depending on the cutoff value employed (Table 3). As AP is currently the major surrogate serological marker of value in the detection of PSC, we evaluated the addition of E3 as a complementary test for the detection of PSC in cases where the diagnosis of PBC and $\mathrm{AIH}$ has been excluded due to negative tests for AMA (Kaplan and Gershwin 2005) and other autoantibodies (Czaja and Manns 2010; Manns and others 2010), respectively (Fig. 6). Only $55 \%$ of PSC patients were positive for AP elevations, whereas $79 \%$ of PSC patients had E3 elevations. Moreover, 97\% of PSC patients exhibited elevations in either circulating AP or E3 strongly supporting the inclusion of E3 testing to improve PSC diagnosis. Such additional testing may possibly also lower the need for invasive liver biopsy (Burak and others 2003) and radiography procedures, which are commonly used to diagnose PSC patients (Angulo and Lindor $1999 b)$. Measuring circulating E3 levels is also of value in diagnosing $\mathrm{AIH}$ and PBC patients since $>84 \%-100 \%$ of these patients had E3 elevations (Table 3).

A combined diagnostic algorithm incorporating E3, the E1/E3 ratio, and CCL22 levels discriminates PSC from healthy controls, $\mathrm{HCV}, \mathrm{PBC}$, and $\mathrm{AIH}$ individuals with a sensitivity of $67 \%-76 \%$ and a specificity of $90 \%-98 \%$ (Table 3 ). Incorporation of IL-15 levels along with E3 and the E1/E3

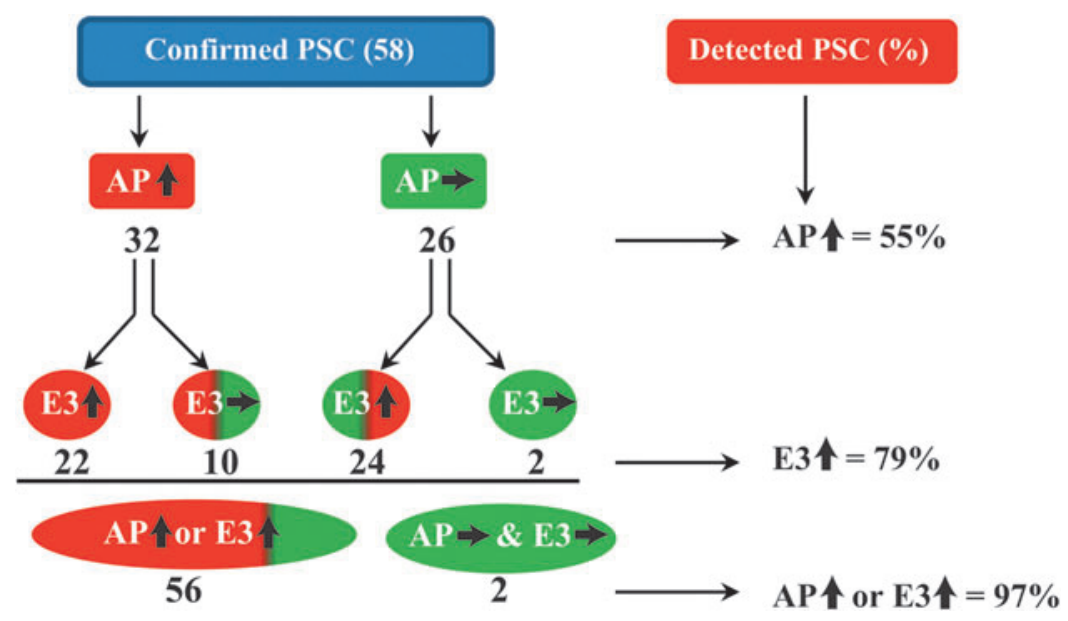

FIG. 6. The value of E3 as an additional surrogate marker to AP in detection of PSC. By combining the elevated value of E3 to the elevated value of $\mathrm{AP}$, the detection rate will increase from $55 \%$ to $97 \%$ for PSC. AP, alkaline phosphatase; AP $\mathbf{t}$, elevated level of $\mathrm{AP} ; \mathrm{AP} \rightarrow$, normal levels of AP; E3 $\mathbf{\uparrow}$, elevated level of E3; E3 $\rightarrow$, normal levels of E3. 


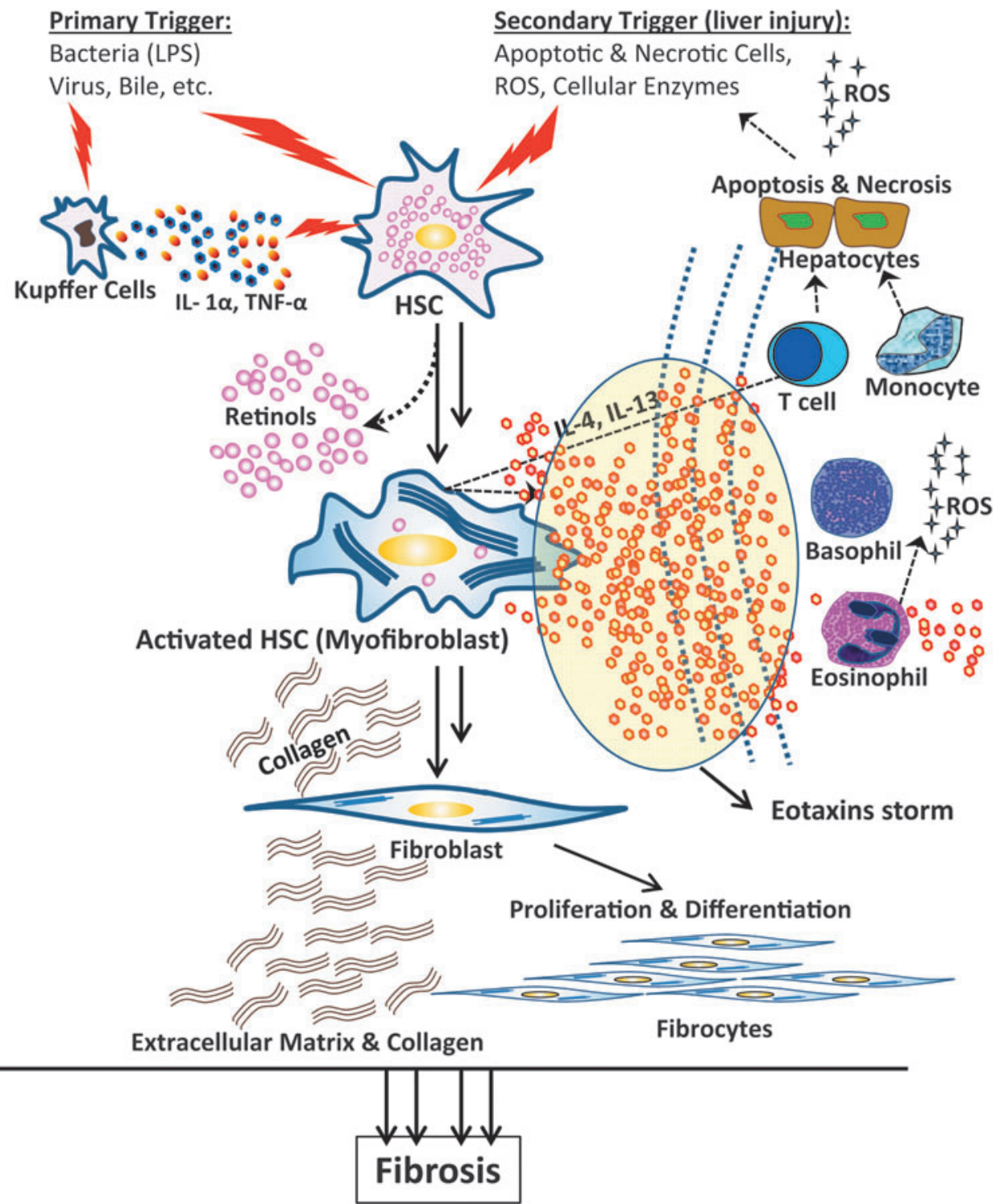

ratio and CCL22 levels was able to diagnose $\mathrm{AIH}$ patients with a sensitivity of $60 \%$ and an excellent specificity of $100 \%$ compared with healthy controls, HCV, and PSC individuals (and with $90 \%$ specificity with respect to PBC patients; Table 3 ).

Although further work is required to validate the role of these biomarkers in future diagnostic procedures, including the determination of these various cytokines/chemokines in PSC, PBC, and AIH patients from around the world and in patients with other liver and other inflammatory diseases, these data suggest that the addition of tests to measure E3, E1, CCL22, and IL-15 could possibly improve the diagnosis of these autoimmune-like liver diseases. It will also be important in the future to evaluate the effect of therapy on the levels of these cytokines/chemokines and to ascertain their prognostic value and ability to predict remission or relapse after cessation of therapy.

Mechanistically, E1 and E3 are Th2-type chemokines that are highly associated with allergic reactions and are mainly chemoattractive for CCR3-expressing eosinophils, basophils, Th2-type T cells, mast cells, and dendritic cells (Gerber and others 1997; Rubbert and others 1998; Ochi and others 1999). They have affinities for other receptors such as CCR1, CCR2,
CCR5, CXCR3, and CX3CR1, through which they can exert a variety of effects. For example, E1 can activate monocytes via CCR5 but block them via CCR2 (Ogilvie and others 2003). It can also antagonize Th1-type effects of CXCL10 by blocking CXCR3 receptor on Th1-type cells (Weng and others 1998). E3 has a 10-fold lower affinity for CCR3 in comparison to E1 (Kitaura and others 1999) and is a natural antagonist for CCR1, CCR2, and CCR5 (Ogilvie and others 2003; Petkovic and others 2004). It has been shown recently that E3 is a functional ligand for CX3CR1, which is a receptor on NK cells, CD8 cells, and monocytes. It could also attract Th1 type cells, which may also contribute to tissue damage (Nakayama and others 2010). This multifunctional role of E1 and E3 indicates that their production may result in attraction of Th1- or Th2-type cells depending on the type and state of disease. Indeed, the diverse infiltration of immune cells into the liver in PSC, PBC, and AIH may indicate a complex and crucial role for eotaxins in the fine-tuning of cellular infiltration occurring at the site of inflammation. This in turn would suggest a differential involvement of liver-resident cells in the pathophysiology of these diseases. In general, the increased levels of E1 and E3 in biological fluids are reported 
to be associated with several allergic diseases, such as allergic asthma, allergic rhinitis, and atopic dermatitis, mainly in the context of eosinophilia (Lilly and others 2001; Terada and others 2001). High levels of E3 also correlate with bullous pemphigoid (Kagami and others 2012), granulomatous vasculitis organ damage in Churg-Strauss syndrome (Polzer and others 2008), and eosinophilic esophagitis, where it has been suggested as a diagnostic biomarker to differentiate the disease from gastroesophageal reflux (Bhattacharya and others 2007). With respect to liver diseases, E1 is elevated in patients with drug-induced liver disease, which appears to be the reason for the recruitment of eosinophils into the liver (Pham and others 2001). The level of E1 in plasma could also be indicative of hepatic inflammation and advanced fibrosis as has been reported in chronic liver diseases, which was correlated with the degree of liver cirrhosis. For this reason, E1 was suggested as an additional biomarker to indicate histological hepatic fibrosis as well as a predictor for an adverse clinical course (Tacke and others 2007). This may agree with our data indicating increased levels of E1 in the PSC group, as PSC is often diagnosed at advanced stages of disease when periductular fibrosis has already resulted in the clinical manifestation of PSC.

Based on the striking elevations of E3 and E1 in PSC and $\mathrm{E} 3$ in $\mathrm{PBC}$ and $\mathrm{AIH}$, we hypothesize an eotaxins-CCR3/ CX3CR1 axis model for the pathophysiology of the liver damage and fibrosis associated with these diseases (Fig. 7). In this model, hepatic stellate cells (HSCs) may be the main producer of eotaxins and play the initial role in the progression of liver fibrosis by attracting different type of immune cells, such as eosinophils, resulting in further production of eotaxins and liver injury in a vicious cycle of disease. HSCs are thought to play a central role in liver fibrosis by losing their retinol droplets and differentiating into activated HSCs or myofibroblasts, then producing large amounts of cytokines/chemokines including eotaxins. They then proliferate, produce extracellular matrix such as collagens, and further differentiate to fibroblasts and fibrocytes, leading to fibrosis (Friedman 2008). As genetic factors have been already described to be associated with autoimmunelike liver diseases, a possibility for other genetic factors with role in the amplification of the production of eotaxins by HSCs may be a subject for further investigation.

This is the first comparative study of cytokine/chemokine levels in the serum of closely related AILD patients from which discriminating diagnostic algorithms have been designed. The observed elevated levels of eosinophilic eotaxins also suggest that these immune mediators may play a key role in the pathogenesis of these challenging liver diseases.

Finally, our findings of abnormal levels of eotaxins in PSC, $\mathrm{PBC}$, and $\mathrm{AIH}$ suggest a new potential therapeutic avenue via abrogating the interaction of E3 and E1 with their receptors such as CX3CR1 and CCR3 using blocking antibodies or drugs. In this regard, an anti-E1 antibody has been used before in an experimental model of AIH (Hegde and others 2011). The use of an anti-E1 antibody has also been proposed as a therapeutic tool in allergic disease (Elsner and others 2004).

\section{Acknowledgments}

We thank Dr. John Elliott (the director of the Alberta Diabetes Institute Molecular Biology Core Laboratory) for introduction and help with the MSD technology. Also, thanks to Dr. Paige Lacy, Dr. Rakesh Bhat, Darren Hockman, and Dr. Michael Logan for helpful inputs.

\section{Author Disclosure Statement}

No competing financial interests exist.

\section{References}

Angulo P, Lindor KD. 1999a. Primary biliary cirrhosis and primary sclerosing cholangitis. Clin Liver Dis 3(3):529-570.

Angulo P, Lindor KD. 1999b. Primary sclerosing cholangitis. Hepatology 30(1):325-332.

Bhattacharya B, Carlsten J, Sabo E, Kethu S, Meitner P, Tavares R, Jakate S, Mangray S, Aswad B, Resnick MB. 2007. Increased expression of eotaxin-3 distinguishes between eosinophilic esophagitis and gastroesophageal reflux disease. Hum pathol 38(12):1744-1753.

Boyer T, Manns MP, Sanyal A. 2011. Zakim and Boyer's hepatology: A textbook of liver disease. Philadelphia (PA): Saunders/ Elsevier. 1408 p.

Burak KW, Angulo P, Lindor KD. 2003. Is there a role for liver biopsy in primary sclerosing cholangitis? Am J Gastroenterol 98(5):1155-1158.

Czaja AJ, Manns MP. 2010. Advances in the diagnosis, pathogenesis, and management of autoimmune hepatitis. Gastroenterology 139(1):58-72.e4.

Elsner J, Escher SE, Forssmann U. 2004. Chemokine receptor antagonists: a novel therapeutic approach in allergic diseases. Allergy 59(12):1243-1258.

Fichorova RN, Richardson-Harman N, Alfano M, Belec L, Carbonneil C, Chen S, Cosentino L, Curtis K, Dezzutti CS, Donoval B, Doncel GF, Donaghay M, Grivel JC, Guzman E, Hayes M, Herold B, Hillier S, Lackman-Smith C, Landay A, Margolis L, Mayer KH, Pasicznyk JM, Pallansch-Cokonis M, Poli G, Reichelderfer P, Roberts P, Rodriguez I, Saidi H, Sassi RR, Shattock R, Cummins JE, Jr. 2008. Biological and technical variables affecting immunoassay recovery of cytokines from human serum and simulated vaginal fluid: a multicenter study. Anal Chem 80(12):4741-4751.

Friedman SL. 2008. Hepatic stellate cells: protean, multifunctional, and enigmatic cells of the liver. Physiol Rev 88(1): 125-172.

Gerber BO, Zanni MP, Uguccioni M, Loetscher M, Mackay CR, Pichler WJ, Yawalkar N, Baggiolini M, Moser B. 1997. Functional expression of the eotaxin receptor CCR3 in $\mathrm{T}$ lymphocytes co-localizing with eosinophils. Curr Biol 7(11): 836-843.

Hegde VL, Nagarkatti PS, Nagarkatti M. 2011. Role of myeloidderived suppressor cells in amelioration of experimental autoimmune hepatitis following activation of TRPV1 receptors by cannabidiol. PLoS One 6(4):e18281.

Kagami S, Kai H, Kakinuma T, Miyagaki T, Kamata M, Sugaya M, Tamaki K, Sato S. 2012. High levels of CCL26 in blister fluid and sera of patients with bullous pemphigoid. J Invest Dermatol 132(1):249-251.

Kaplan MM, Gershwin ME. 2005. Primary biliary cirrhosis. N Engl J Med 353(12):1261-1273.

Kitaura M, Suzuki N, Imai T, Takagi S, Suzuki R, Nakajima T, Hirai K, Nomiyama H, Yoshie O. 1999. Molecular cloning of a novel human CC chemokine (Eotaxin-3) that is a functional ligand of CC chemokine receptor 3. J Biol Chem 274(39): 27975-27980.

Lilly CM, Nakamura H, Belostotsky OI, Haley KJ, GarciaZepeda EA, Luster AD, Israel E. 2001. Eotaxin expression after 
segmental allergen challenge in subjects with atopic asthma. Am J Respir Crit Care Med 163(7):1669-1675.

Lohse AW, Mieli-Vergani G. 2011. Autoimmune hepatitis. J Hepatol 55(1):171-182.

Manns MP, Czaja AJ, Gorham JD, Krawitt EL, Mieli-Vergani G, Vergani D, Vierling JM. 2010. Diagnosis and management of autoimmune hepatitis. Hepatology 51(6):2193-2213.

Mason AL. 2011. The evidence supports a viral aetiology for primary biliary cirrhosis. J Hepatol 54(6):1312-1314.

Minuk GY, Rascanin N, Paul RW, Lee PW, Buchan K, Kelly JK. 1987. Reovirus type 3 infection in patients with primary biliary cirrhosis and primary sclerosing cholangitis. J Hepatol 5(1):8-13.

Nakayama T, Watanabe Y, Oiso N, Higuchi T, Shigeta A, Mizuguchi N, Katou F, Hashimoto K, Kawada A, Yoshie O. 2010. Eotaxin-3/CC chemokine ligand 26 is a functional ligand for CX3CR1. J Immunol 185(11):6472-6479.

Ochi H, Hirani WM, Yuan Q, Friend DS, Austen KF, Boyce JA. 1999. T helper cell type 2 cytokine-mediated comitogenic responses and CCR3 expression during differentiation of human mast cells in vitro. J Exp Med 190(2): 267-280.

Ogilvie P, Paoletti S, Clark-Lewis I, Uguccioni M. 2003. Eotaxin3 is a natural antagonist for CCR2 and exerts a repulsive effect on human monocytes. Blood 102(3):789-794.

Petkovic V, Moghini C, Paoletti S, Uguccioni M, Gerber B. 2004. Eotaxin-3/CCL26 is a natural antagonist for CC chemokine receptors 1 and 5 . A human chemokine with a regulatory role. J Biol Chem 279(22):23357-23363.

Pham BN, Bemuau J, Durand F, Sauvanet A, Degott C, Prin L, Janin A. 2001. Eotaxin expression and eosinophil infiltrate in the liver of patients with drug-induced liver disease. J Hepatol 34(4):537-547.

Polzer K, Karonitsch T, Neumann T, Eger G, Haberler C, Soleiman A, Hellmich B, Csernok E, Distler J, Manger B, Redlich K, Schett G, Zwerina J. 2008. Eotaxin-3 is involved in ChurgStrauss syndrome-a serum marker closely correlating with disease activity. Rheumatology 47(6):804-808.

Ranson T, Vosshenrich CA, Corcuff E, Richard O, Muller W, Di Santo JP. 2003. IL-15 is an essential mediator of peripheral NK-cell homeostasis. Blood 101(12):4887-4893.

Rubbert A, Combadiere C, Ostrowski M, Arthos J, Dybul M, Machado E, Cohn MA, Hoxie JA, Murphy PM, Fauci AS, Weissman D. 1998. Dendritic cells express multiple chemokine receptors used as coreceptors for HIV entry. J Immunol 160(8):3933-3941.
Tacke F, Trautwein C, Yagmur E, Hellerbrand C, Wiest R, Brenner DA, Schnabl B. 2007. Up-regulated eotaxin plasma levels in chronic liver disease patients indicate hepatic inflammation, advanced fibrosis and adverse clinical course. J Gastroenterol Hepatol 22(8):1256-1264.

Terada N, Hamano N, Kim WJ, Hirai K, Nakajima T, Yamada H, Kawasaki H, Yamashita T, Kishi H, Nomura T, Numata T, Yoshie O, Konno A. 2001. The kinetics of allergen-induced eotaxin level in nasal lavage fluid: its key role in eosinophil recruitment in nasal mucosa. Am J Respir Crit Care Med 164(4):575-579.

Tischendorf JJ, Hecker H, Kruger M, Manns MP, Meier PN. 2007. Characterization, outcome, and prognosis in 273 patients with primary sclerosing cholangitis: a single center study. Am J Gastroenterol 102(1):107-114.

Vento S, Cainelli F. 2004. Is there a role for viruses in triggering autoimmune hepatitis? Autoimmun Rev 3(1):61-69.

Weng Y, Siciliano SJ, Waldburger KE, Sirotina-Meisher A, Staruch MJ, Daugherty BL, Gould SL, Springer MS, DeMartino JA. 1998. Binding and functional properties of recombinant and endogenous CXCR3 chemokine receptors. J Biol Chem 273(29):18288-18291.

Address correspondence to: Dr. Abdolamir Landi Department of Medical Microbiology and Immunology Li Ka Shing Institute of Virology University of Alberta

6-010 Katz Group-Rexall Centre for Health Research Edmonton T6G 2E1

Canada

E-mail: landi@ualberta.ca

Prof. Michael Houghton Department of Medical Microbiology and Immunology Li Ka Shing Institute of Virology University of Alberta 6-010 Katz Group-Rexall Centre for Health Research Edmonton T6G 2E1

Canada

E-mail: michael.houghton@ualberta.ca

Received 25 July 2013/Accepted 10 September 2013 